Int. J. Dev. Biol. 54: 1509-1516 (2010)

doi: $10.1387 / \mathrm{ijdb} .103139 \mathrm{dk}$

\title{
The expression of teneurin-4 in the avian embryo: potential roles in patterning of the limb and nervous system
}

\author{
DANIELA KENZELMANN BROZ1,\#, RICHARD P. TUCKER ${ }^{1,2, \#, ~ N A T H A N I E L ~ T . ~ L E A C H M A N ² ~}$ \\ and RUTH CHIQUET-EHRISMANN*,1 \\ ${ }^{1}$ Friedrich Miescher Institute, Novartis Research Foundation, Basel, Switzerland and \\ ${ }^{2}$ Department of Cell Biology and Human Anatomy, University of California at Davis, Davis, CA USA
}

\begin{abstract}
Teneurins are type II transmembrane proteins that play important roles in pattern formation in Drosophila, axon fasciculation and organogenesis in Caenorhabidits elegans, and neuronal pathfinding in the visual system of the mouse. There is evidence that a peptide derived from a proteolytic event near the C-terminus of teneurins leads to formation of an active neuropeptide, while processing at and near the transmembrane domain leads to shedding of the extracellular domain into the extracellular matrix and the generation of an intracellular fragment that is transported to the nucleus. In vertebrates there are four teneurins. Here, we have studied the expression of teneurin-4 in the chicken embryo. An antiserum against part of the intracellular domain of teneurin-4 recognizes several low molecular weight bands on immunoblots of embryonic chicken brain homogenates, indicating that teneurin-4 is likely to be processed at one or more predicted proteolytic cleavage sites. Antisera against the EGF-like repeats of the extracellular domain label some mesenchyme in the early embryo, and near basement membranes this labeling partially overlaps with anti-laminin (gamma 1 chain) immunostaining. At embryonic day 7, anti-teneurin-4 labels bundles of axons in the nasal, but not temporal retina. Later in development, retinal expression switches so that teneurin-4 is found in the temporal, but not nasal, ganglion cell layer. Teneurin-4 immunolocalization was also compared with other teneurins in the developing limb, where each teneurin is expressed in distinctive regions. These patterns of expression suggest roles for teneurin-4 in patterning and neuronal pathfinding in the avian embryo.
\end{abstract}

KEY WORDS: development, limb bud, retina, Odz, Ten-m

Teneurins are large (approximately $300 \mathrm{kDa}$ ) type-2 transmembrane glycoproteins composed of an intracellular domain (ICD) and an extracellular domain (ECD) with tenascin-type EGF-like repeats and tyrosine-rich YD repeats. One of the first teneurins to be characterized, $\operatorname{ten}^{\mathrm{m}}$ (also known as odz), was found in Drosophila, where it was shown to act as a pair-rule gene (Baumgartner et al. 1994; Levine et al. 1994). Ten ${ }^{\mathrm{m}}$ is also expressed in the central nervous system (CNS) of Drosophila (Levine et al. 1997; Minet et al. 1999), as is the related protein Ten ${ }^{a}$ (Fascetti and Baumgartner, 2002; Rakovitsky et al. 2007). The single teneurin of $C$. elegans, ten-1, is required for the normal development of the gonad and the fasciculation of subsets of axons (Drabikowski et al. 2005; Trzebiatowska et al. 2008). In vertebrates there are four teneurins numbered 1-4. These are primarily found in the CNS where they have been shown to be expressed by interconnected populations of neurons as well as in functionally relevant gradients (see reviews by Tucker et al. 2007; Young and Leamey, 2009). The potential importance of teneurins in neuronal patterning is illustrated by the teneurin- 3 knockout mouse, which has

\footnotetext{
Abbreviations used in this paper: ICD, intracellular domain; ECD, extracellular domain; CNS, central nervous system; E, embryonic day; PBS, phosphate buffered saline
}

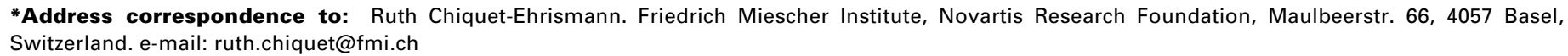


abnormal pathfinding of axons at the optic chiasm resulting in the loss of binocular vision (Leamey et al. 2007).

$T e n^{m}$ was the first pair-rule gene to be characterized that encoded a transmembrane protein; the other pair-rule genes were transcription factors. Teneurins may be transcription factors as well, as antibodies to the ICD of $C$. elegans ten- 1 immunolabel cell nuclei, while antibodies to the ECD label sites of cell-cell adhesion (Drabikowski et al. 2005). Similar observations have come from vertebrate systems. When the ICD of teneurin-1 (Nunes et al. 2005) or teneurin-2 (Bagutti et al.2003) is expressed in vitro it localizes to the cell nucleus, and antibodies to the ICD of avian teneurin-1 label some cell nuclei in a subset of tissues stained by antibodies to the ECD (Kenzelmann et al. 2008). When a potential nuclear localization signal in the ICD of teneurin-1 is mutated, the ICD fails to translocate to the nucleus (Kenzelmann et al. 2008). However, genes that are regulated by the ICD of teneurins have yet to be identified.

Here we have studied the expression of teneurin-4 and initiated a study of its processing in the chicken embryo. Novel antisera raised to portions of the ICD and ECD of the avian protein were used to study its expression both by immunoblotting and immunohistochemistry, and its expression was compared with that of teneurins $-1,-2$ and -3 . Detailed examination of limbs and the retina show that each of the teneurins is expressed in different patterns in these tissues, and confirms that teneurins are likely to play fundamental roles in patterning both in the CNS and in nonneuronal tissues during vertebrate development.

\section{Results}

\section{Evidence of teneurin-4 processing}

Other teneurins can be processed by proteolytic cleavage between the transmembrane domain and the tenascin-type EGFlike repeats, which may be the first step leading to release of the ICD to translocate to the nucleus (e.g., Kenzelmann et al. 2008). To see if teneurin- 4 is similarly processed, antisera were raised against the ICD and the EGF-like repeats found in the ECD of

Fig. 1. Immunoblots with anti-teneurin-4 antibodies. (A) A stick diagram showing the domain organization of teneurin-4 and the approximate composition of the expressed proteins used to generate antisera to the intracellular domain (ICD) and extracellular domain. The arrowhead indicates a potential furin cleavage site (see below). (B) Homogenates of embryonic day 17 telencephalon (TEL), retina (RET), optic tectum (OT) and cerebellum (CBL) were separated by SDS-PAGE, transferred to filter and probed with the anti-ICD and anti-EGF sera. The anti-ICD recognizes a band running at approximately $64 \mathrm{kDa}$ (arrowhead), suggesting that teneurin-4 is processed at a putative furin cleavage site between the transmembrane domain and the EGF-like repeats. Smaller bands (asterisks) may represent further processing or non-specific immunostaining. The anti-EGF recognizes a single band running higher than the $182 \mathrm{kDa}$ size standard. (C) Homogenates of E7 and E17 retina (RET) and cerebellum (CBL) were separated by electrophoresis, transferred and probed with antisera to the teneurin-4 intracellular domain (ICD) or EGF repeats. Anti-ICD recognizes a single band running at approximately $300 \mathrm{kDa}$ in homogenates of the $E 7$ retina that is visible after long exposures. The anti-EGF serum shows that the relative levels of teneurin- 4 expression are higher in both the retina and cerebellum and $E 7$ than at E17. The single band seen in Fig. 1B can be resolved into an apparent doublet (double arrows), with the lower band running near 230-250 kDa, and the upper band running at approximately 280-300 kDa. teneurin-4 (Fig. 1A) and used on immunoblots of homogenates of developing chicken brain. The teneurin-4 ICD antiserum recognized a prominent band at approximately $64 \mathrm{kDa}$ (arrowhead, Fig. 1B) as well as three smaller bands with apparently molecular weights between approximately 30 and 50 kDa (asterisks, Fig. 1B). Longer exposures also revealed a high molecular weight band similar to the single, high molecular weight band (280-300 $\mathrm{kDa}$ ) recognized by teneurin-4 EGF antiserum (arrowhead, Fig. 1C). The low molecular weight bands recognized by anti-teneurin4 ICD are evidence that teneurin- 4 may be processed by proteolytic cleavage; the single high molecular weight band recognized by anti-teneurin-4 EGF is evidence of the specificity of the antiserum for teneurin-4. Note that an anti-EGF serum raised in a second rabbit recognized the same high molecular weight bands, but more faintly. No significant differences (except in the intensity of the bands) were seen between the different brain regions examined.

Homogenates of different CNS regions isolated from embryos at different stages were also studied by immunoblotting. In both the retina and the cerebellum the intensity of the bands recognized by the anti-EGF serum diminished markedly between E7 and E17 (Fig. 1C). High molecular weight proteins were better resolved on this gel than in Fig. 1B. This immunoblot reveals that what appeared to be a single band that was recognized in Fig. 1B

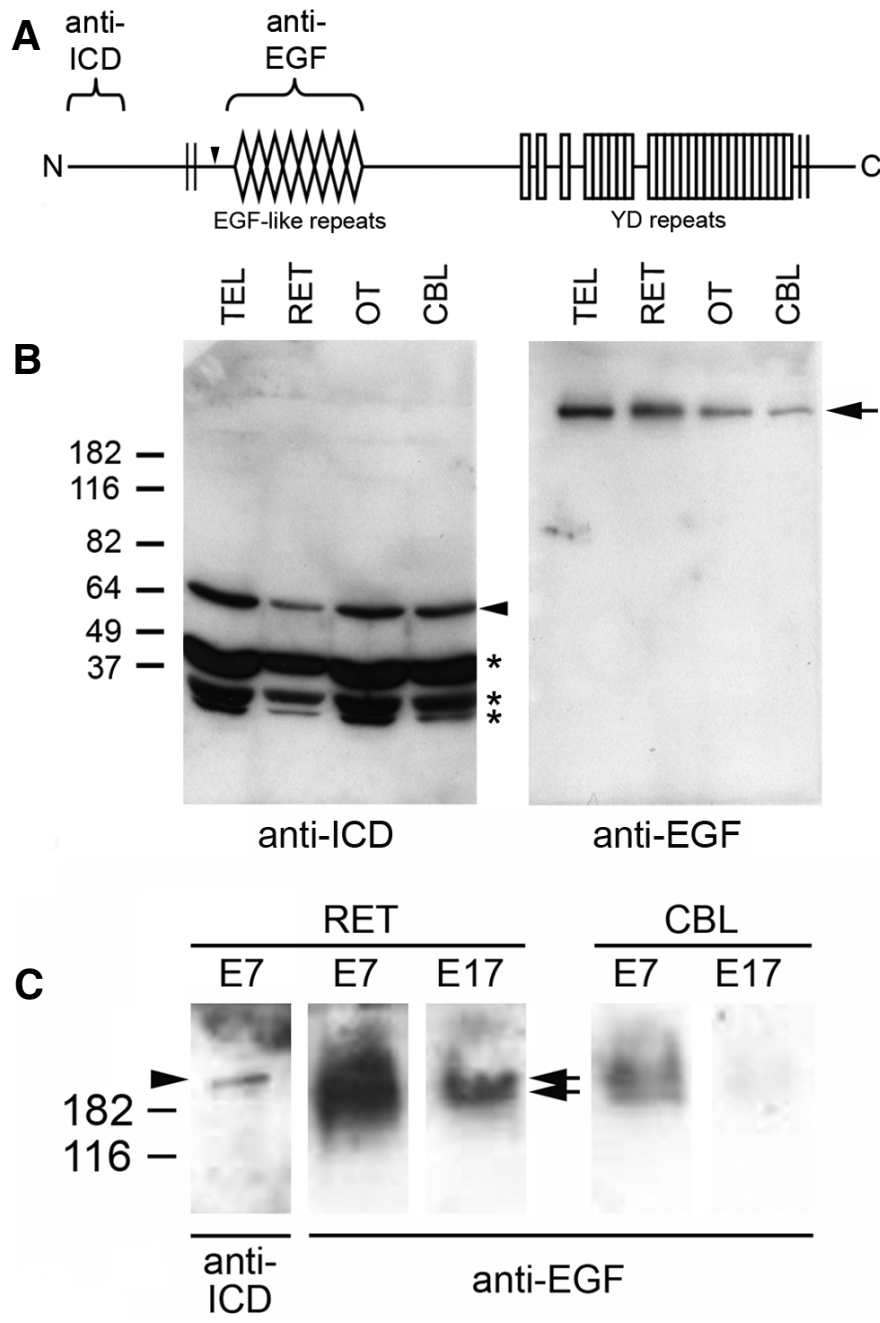


by the teneurin-4 EGF antiserum is a doublet, with the lower band running at $230-250 \mathrm{kDa}$ and the larger band running at $280-300 \mathrm{kDa}$ (sizes were estimated by extrapolation from the positions of the standards). Fig. 1C also shows that the anti-ICD recognizes a single high molecular weight band (and not a doublet) when exposed for longer periods.

\section{Immunolocalization of teneurin-4 in the chick embryo}

Since the anti-teneurin-4 EGF sera gave an unambiguous, specific staining pattern on immunoblots it was used to determine the location of teneurin-4 expression during avian development. At embryonic day $(E)$ 3.5, immunostaining was observed in the sclerotome, mesenchyme surrounding the dorsal aorta (not shown) and gut (Fig. 2A). The rest of the embryo was unstained. In the gut, the teneurin-4 immunostaining was seen surrounding the endoderm and nests of cells found in the surrounding mesenchyme. Secondary antibody alone did not label this tissue (Fig. 2B). The pattern of teneurin-4 expression resembles that of laminin (Fig. 2C), and high magnification analysis of double label immunohistochemistry with antibodies to laminin and teneurin-4 show that some, but not all, of the teneurin-4 immunostaining overlaps with laminin (Fig. 2D). At E4, the most prominent immunolabeling was seen in the mesenchyme surrounding the growing lung bud and the adjacent epithelium (Fig. 2E). The mesenchyme around the esophagus is unlabeled. Preimmune serum did not label the lung bud mesenchyme (Fig. 2F). Another prominent site of teneurin-4 expression at E4 is the developing limb. At E4 teneurin-4 immunostaining is strong in the distal mesenchyme of the anterior limb (Fig. 2G; note that in the posterior limb this mesenchyme is unstained [see below for details]). Anti-teneurin4 also labeled the mesenchyme surrounding the developing eye at this stage (Fig. $2 \mathrm{H}$ ) and at E7 and E12. The latter is described with the description of the immunolabeling of the retina below. The teneurin-4 antibody also labels lung mesenchyme at later stages of development (Fig. 2l) as well as the mesenchyme of the midgut at E17 (Fig. 2J), but not the mesenchyme of proventriculus (not shown). Anti-teneurin-4 immunostaining was not observed in the heart at earlier stages, but at E17 the antibody labeled the inner lining (facing the ventricular chamber) of the atrioventricular valves (Fig. $2 \mathrm{~K}$ ). The epicardium was also faintly stained at this stage, as were coronary vessels (Fig. 2L). Striated muscle was not stained with the teneurin-4 antibody at any stage examined.

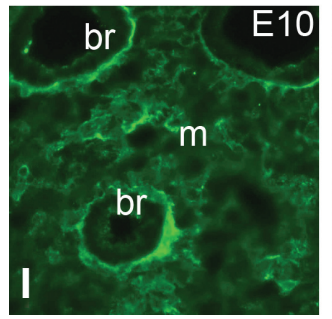

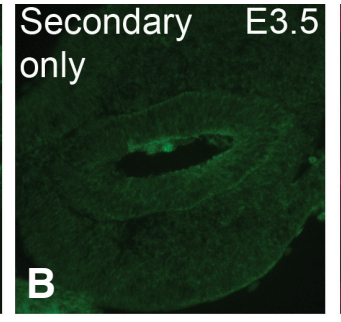
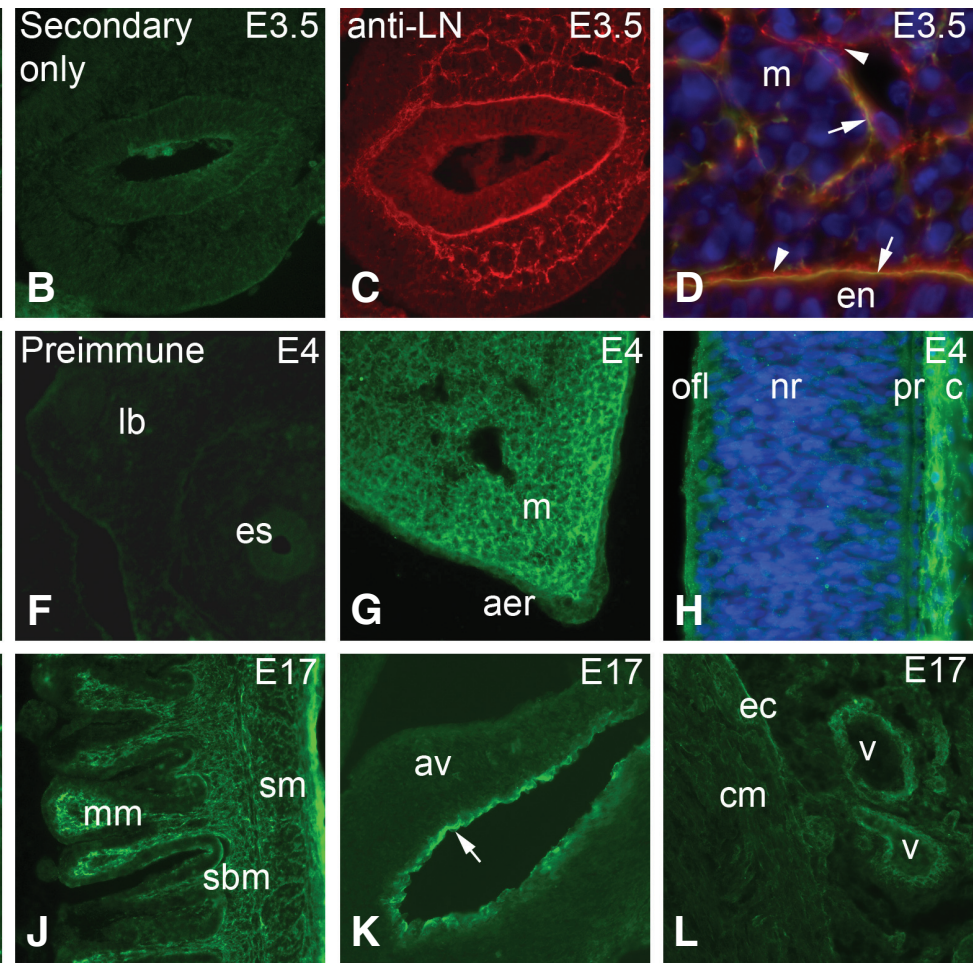

Fig. 2. Immunolocalization of teneurin-4 in non-neuronal tissues. (A,B) At $E 3.5$ the anti-EGF immunolabels the area surrounding the endoderm (en) of the developing distal foregut as well as around (gamma 1) gives a similar staining pattern. (D) When merged and viewed at higher magnification areas the 4 retina. The neural retina (nr) and pigment retina (pr) are unstained. Periocular connective tissue (c) (br) and in the mesenchyme. (J) The anti-EGF also stains the muscularis mucosa $(\mathrm{mm})$ in the villi of the anti-EGF immunostains the inner lining (arrow) of the atrioventricular valves (av). Cardiac muscle (cm) is unstained, but there is faint labeling of the epicardium (ec) and coronary vessels (v).

Teneurin-4 was also detected by immunohistochemistry in the developing CNS. At E4, there was faint immunostaining with the anti-teneurin-4 in the optic fiber layer of the retina (Fig. $2 \mathrm{H}$ ) and in the diencephalon near the optic stalk (Fig. 3A). Teneurin-4 immunoreactivity was seen some, but not all, of the axons found in the marginal layer in this region. By E7 expression in the CNS was limited to what appear to be bundles of axons in the optic fiber layer of the nasal retina (Fig. $3 \mathrm{C}$ ), but not the temporal retina (Fig. 3B). The periocular mesenchyme is also prominently labeled at this stage. By E10 the optic fiber layer of the occipital part of optic tectum, to which processes from the nasal retina project, is also positively immunostained with the anti-teneurin-4 (Fig. 3D). Other parts of the optic tectum are unlabelled. At E12 there was faint teneurin-4 immunoreactivity in the optic nerve, but in the optic tract the immunostaining was bright and heterogeneous (Fig. 3E). In the optic tectum there was bright labeling in the optic fiber layer, but not elsewhere (Fig. 3F). By E17 the chicken brain resembles the brain of the adult, and the pattern of teneurin- 4 immunostaining can be 
interpreted more precisely. One of the strongest sites of expression was in the fiber tract connecting the hippocampus with the lateral habenular nucleus (Fig. 3G). In the hindbrain several nuclei were positive for teneurin-4. Interestingly, these were only partially immunostained. For example, the lateral parts of the nucleus laminaris and nucleus magnocellularis are strongly labeled, but not the medial parts (Fig. $3 \mathrm{H})$. There was also a gradient of expression in the retina: in more temporal parts of the retina the teneurin- 4 antiserum labeled neurons in the retinal ganglion cell layer strongly (Fig. 3I). The staining was punctate and strongest in the perikarya and proximal dendrites. In contrast, the nasal retina is unlabeled (Fig. $3 \mathrm{~J}$ ). In the optic tectum the optic fiber layer was labeled at E17, and the outer part of the stratum griseum et fibrosum superficiale was labeled in some regions, but not all (Fig. 3K). Elsewhere in the mesencephalon (Fig. $3 \mathrm{~L}$ ) the nucleus isthmus pars magnocellularis, which connects to the outer layers of the optic tectum (e.g., Tombol et al. 2006), is labeled, as is part of the nucleus isthmus pars parvocellularis. The nucleus mesencephalicus lateralis pars dorsalis is also labeled. In the E17 cerebellum, which is a prominent site of expression for other teneurins (e.g., Kenzelmann et al. 2008), teneurin-4 immunostaining is faint (not shown). This corresponds with the faint band seen in homogenates of the E17 cerebellum (Fig. 1C).

The nasotemporal differences in expression seen in the retina at $E 7$ and $E 17$ were also seen at E12 (Fig. 4). In a low magnification overview of the orbit one sees prominent antiteneurin-4 immunostaining in the nasal retina. Higher magnification reveals that this is due to the bright labeling of the optic fiber layer in this region. In contrast, the cell bodies of retinal ganglion cells are stained more strongly in the temporal region than in the nasal region. Three laminae within the inner plexiform layer are immunostained with the anti-teneurin-4 in the temporal retina (see below), but only two are labeled in the nasal retina. The choroid and loose connective tissue external to the sclera are stained throughout the orbit.

\section{Teneurin-4 expression compared with the expression of other teneurins}

Because of its prominent expression in developing limbs and the developing visual system, teneurin-4 expression was compared side-by-side in these regions with the expression of the other teneurins.

In the E4 limb each of the teneurins had a distinctive pattern of expression. Teneurin-1 was found in the dorsal ectoderm as far as the apical ectodermal ridge, but in the ventral limb teneurin-1 expression was found in the mesenchyme (Fig. 5A). An identical pattern was seen with a second teneurin-1 antibody raised against the ICD, but this antiserum stained nuclei (Fig. 5A, inset). As described previously (Tucker et al. 2000), teneurin-2 expression in the limb was limited to the apical ectoectodermal ridge (Fig. 5B). Teneurin-3 was concentrated in

Fig. 3. Immunolocalization of teneurin-4 in neuronal tissues during development. (A) At E4 a patch of axons within the marginal layer ( $\mathrm{ml}$; arrow) of the diencephaIon (di) are labeled. Other axons are unlabeled (arrowhead). V, ventricle. (B,C) At E7 the connective tissue (c) surrounding the eyes is brightly stained. The pigment retina (pr) and neural retina (nr) are unlabeled, except for bundles of axons in the optic fiber layer of the nasal retina (arrows). The optic fiber layer of the temporal retina (arrowhead) is unlabeled. (D) At E10 the optic fiber layer (ofl) of the posterior optic tectum is immunostained with anti-EGF. Deeper layers (e.g., the stratum griseum fibrosum et superficiale [sgfs]) are unlabeled. (E,F) At E12 the anti-EGF labels the optic chiasm (co). Within the optic tract (tro) the label is brightest laterally; labeling of the optic nerve (CNII) is faint. In the posterior optic tectum the optic fiber layer is labeled, but not deeper layers. sgc, stratum griseum centrale; sgp, stratum griseum periventriculare. (G) At E17 a fiber tract running near the lateral habenular nucleus (hl) is immunostained by the anti-EGF (ar-
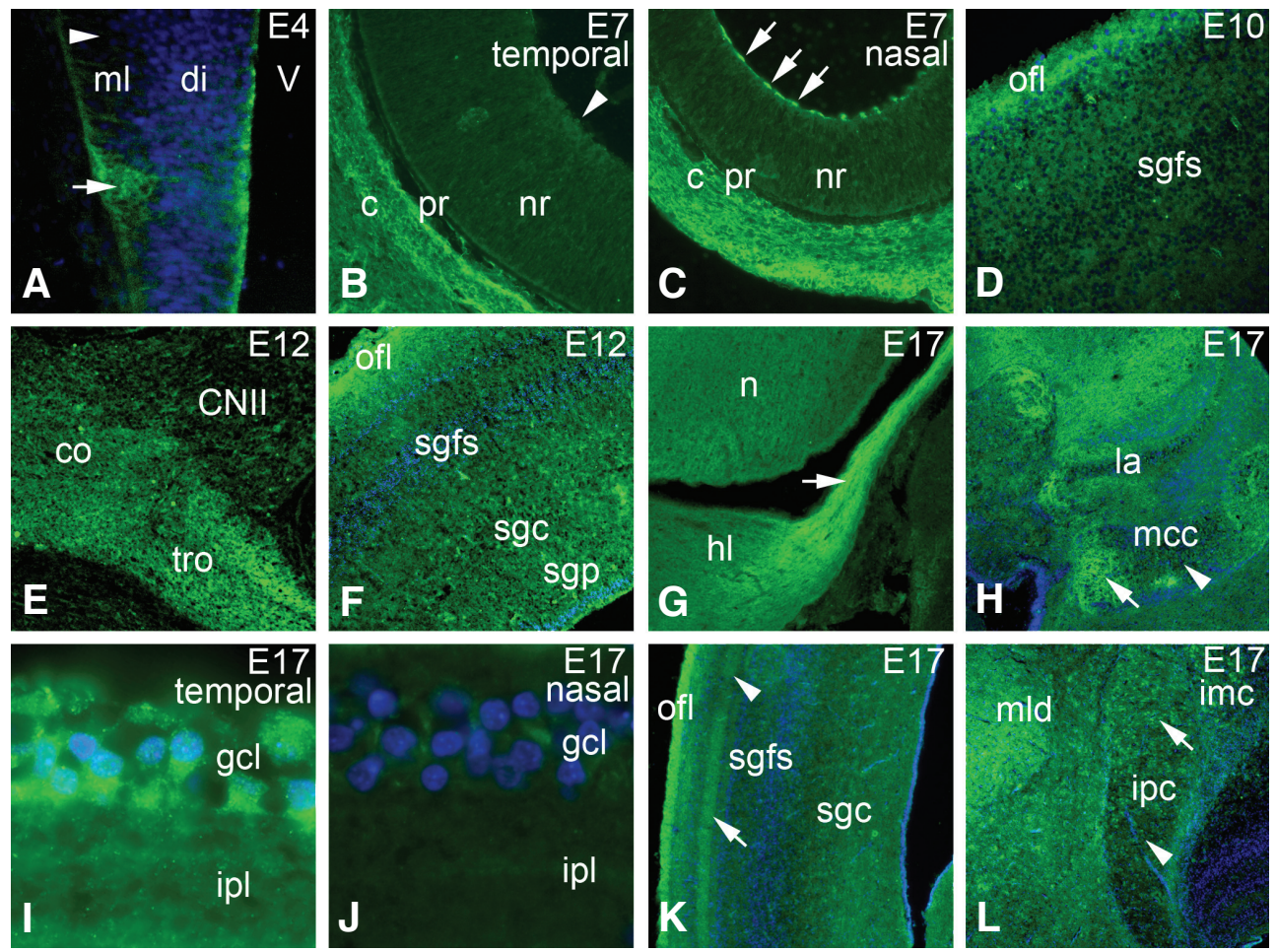

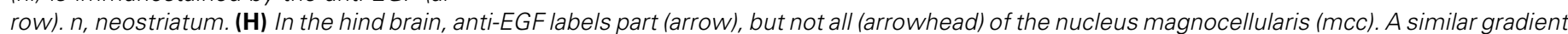

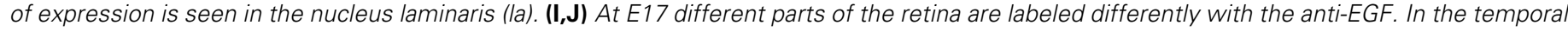

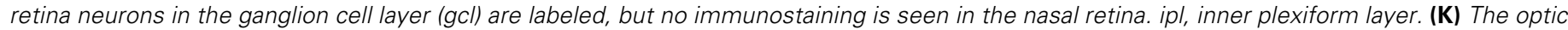

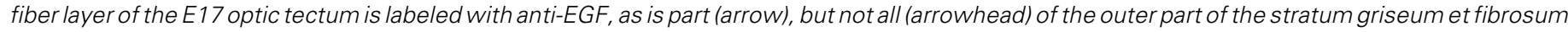

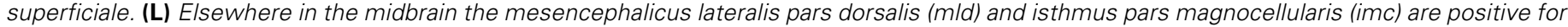
teneurin-4, as is part (arrow), but not all (arrowhead) of the nucleus isthmus pars parvocellularis (ipc). 
Fig. 4 (Right). Teneurin-4 expression in the E12 orbit. The anti-EGF teneurin-4 serum immunolabels the optic fiber layer (ofl) in the nasal retina (black and white arrow in the inset 'nasal'). The ganglion cell layer just deep to the optic fiber layer is unstained, but there are two laminae (arrows, inset 'nasal') that are positive for teneurin-4 in the inner plexiform layer. In contrast, the optic fiber layer of the temporal retina is unlabelled (arrowhead, inset 'temporal'). The ganglion cell layer (gcl) and three laminae in the inner plexiform layer (arrows, inset 'temporal') are positive for teneurin-4 expression. Loose connective tissue (c) external to the sclera and the choroid are also stained. In the low magnification overview note the position of the nasal septum (ns) and a nasal turbinate (t) as well as the diencephalon (di) near the optic chiasm.

the dorsal limb, where it was found in both the ectoderm and the underlying mesenchyme (Fig. 5C). As described above, the teneurin-4 immunostaining pattern changed as one went through the limb from anterior (rostral) to posterior (caudal): in the anterior limb teneurin-4 was found in the distal mesenschyme (as described earlier), but in the posterior limb teneurin-4 was in the apical ectodermal ridge and the proximal mesenchyme (Fig. 5D). Thus, teneurins are expressed in patterns that may reflect roles in the development of dorso-ventral, anteriorposterior, and proximal-distal features of the limbs. Note that the immunostaining of the developing limbs was transient: the anti-teneurin-4 did not stain limb buds prior to E3, and the staining of the limbs is lost between E4 and E5.

We previously described the expression of teneurin-1 and 2 in the E17 central retina (Kenzelmann et al. 2008): teneurin1 is found in the retinal ganglion cell layer, inner plexiform layer and outer nuclear layer, and within the inner plexiform layer it is most prominently expressed in two laminae (Fig. 5E); teneurin2 is found in the inner plexiform layer and the outer plexiform

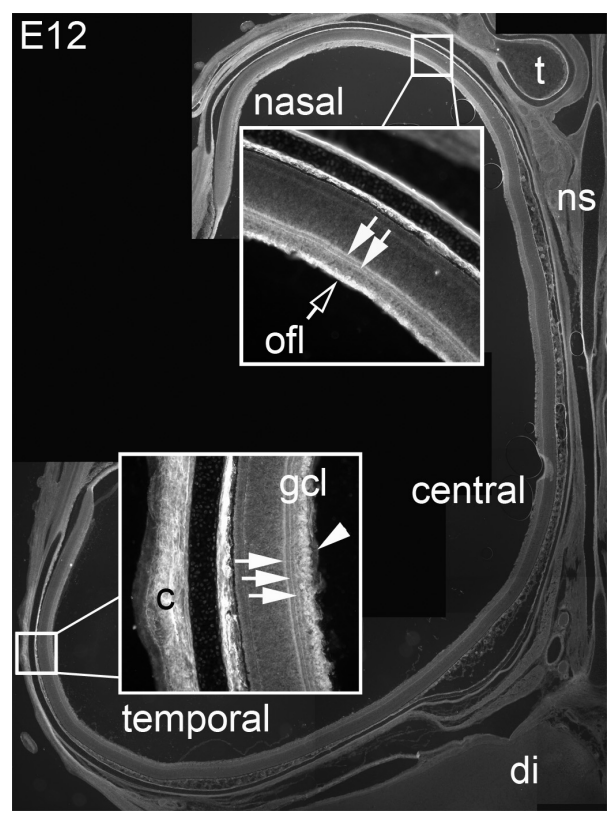

layer, and within the inner plexiform layer it is found in laminae that are distinct from the strata expressing teneurin-1 (Fig. 5F). Teneurin-3 is expressed in a pattern that resembles teneurin1 , but with yet a third laminar pattern (Fig. 5G). Finally, in the central retina teneurin-4 is limited to the inner plexiform layer, where it is expressed faintly in two outer laminae and strongly in the outermost lamina adjacent to the inner nuclear layer (Fig. $5 \mathrm{H}$ ). This pattern is distinctive from the prominent labeling of the ganglion cell layer seen in the temporal retina and the absence of staining in the nasal retina (described earlier).
Fig. 5. Teneurin expression in the limb and central retina. (A)

Teneurin-1 is expressed in the dorsal ectoderm (de) of the E4 limb, as well as in the ventral mesenchyme (vm). A second teneurin-1 antiserum raised against an intracellular domain epitope gives the same staining pattern, but is nuclear (inset). (B) Teneurin-2 immunoreactivity is limited to the apical ectodermal ridge (aer, arrow). (C) Teneurin-3 immunostaining is confined to the dorsal ectoderm and dorsal mesenchyme (dm). (D) As shown in Fig. 2G, teneurin-4 immunoreactivity is found in the mesenchyme of the anterior limb. In the central limb (shown here) the expression is seen in the apical ectoderm ridge. Teneurin4 is also expressed in the proximal mesenchyme (pm) of the limb (inset). (E) In the central retina at E17, teneurin-1 is found in the ganglion cell layer (gcl), inner nuclear layer (inl) and outer nuclear layer (onl). Two

laminae are labeled in the inner plexiform layer (ipl, arrows). (F) Teneurin-2 is expressed in three laminae in the inner plexiform layer (arrows) and in the outer plexiform layer (op/). (G) Teneurin-3 is found throughout the retina as well as in three laminae (arrows) in the inner plexiform layer that are different from the laminae recognized by the anti-teneurin-2. (H) In the central retina (but not the temporal or nasal retina, see Fig. 3l,J), teneurin-4 is expressed faintly in two laminae of the inner plexiform layer, and more strongly in the lamina that lies adjacent to the inner nuclear layer (arrows).

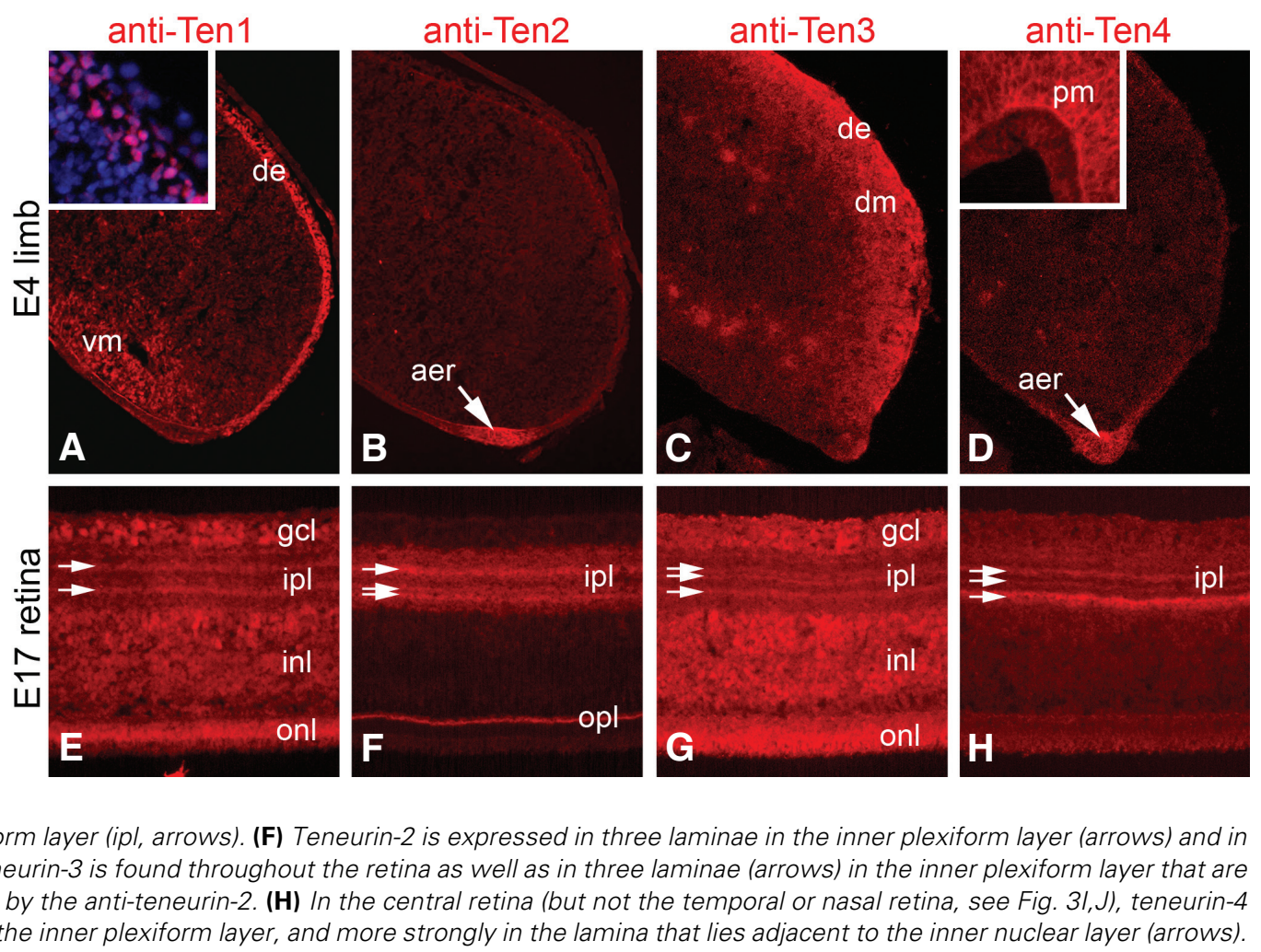




\section{Discussion}

We previously used antisera raised against the ICD and EGFlike repeats of teneurin-1 on immunoblots of embryonic chicken brain (Kenzelmann et al. 2008). The anti-teneurin-1 ICD antibodies recognized a band running at approximately $64 \mathrm{kDa}$, and the antiserum directed to the EGF-like repeats of teneurin-1 recognized larger bands corresponding to the full length protein. Here, antisera raised against similar portions of teneurin-4 produced similar patterns on immunoblots, suggesting that teneurin-4 may be similarly processed. For teneurin-2 (Rubin et al. 1999) and teneurin-3 (Leachman and Tucker, unpublished observations), the primary proteolytic processing appears to take place at a furin cleavage site found between the transmembrane domain and the first EGF-like repeat. This would generate a product with the theoretical molecular weight of approximately $55 \mathrm{kDa}$ (these products have larger apparent molecular weights when analyzed by SDS-PAGE, see Nunes et al. 2005). Teneurin-4 has the motif PRQSR at the site corresponding to the furin cleavage sites of teneurin- 2 and -3 that would generate a product with a theoretical molecular weight of $57 \mathrm{kDa}$. This may correspond to the band seen at $65 \mathrm{kDa}$ on immunoblots (Fig. 1B). The smaller products seen with the anti-teneurin-4 ICD may represent products generated through additional intracellular processing (faint bands of this size are also seen with the anti-teneurin-1 ICD; Kenzelmann et al. 2008), or they could represent products generated by proteolytic cleavage at other extracellular sites. In addition to the putative furin site there are multiple dibasic residues that are potential sites of proteolytic cleavage (Rholam and Fahy, 2009) in the region found just outside the transmembrane domain of teneurin-4 that have the potential to generate multiple products with theoretical molecular weights ranging from 45 to $55 \mathrm{kDa}$. However, it is also possible that these smaller bands correspond to a protein or proteins that are unrelated to teneurin-4 yet are recognized by the antiserum. For that reason, only the antibody to the EGF-like repeats, which recognized a single band on the immunoblots and recognized tissues where teneurin-4 mRNAs were identified previously at E3.5 and E4 (see below), was used for the immunohistochemistry in the figures. However, we did process many slides with the anti-ICD and nuclear staining was never observed. This leads us to suggest that if the ICD of teneurin-4 is processed only a small fraction, if any, may enter the nucleus. Note that there is also considerable evidence that the $\mathrm{C}$ terminus of teneurins can be processed to produce functionally relevant neuropeptides (reviewed by Lovejoy et al. 2009); this processing was not studied here.

Previously we described the expression of teneurin-4 in the E3.5 and E4 chicken by whole mount in situ hybridization and demonstrated that it was expressed in periocular mesenchyme and in the distal mesenchyme of the anterior, but not posterior, limb (Tucker et al. 2000). We also showed that it was found in mesenchyme in the proximal limb and in a limited part of the diencephalon. Our immunostaining both confirms and expands upon these previously published results, which for technical reasons were limited to superficial tissues at early stages of development. The antiserum to the EGF-like repeats of teneurin4 recognized periocular mesenchyme (Fig. 2H; Fig. $3 \mathrm{~B}, \mathrm{C}$ ), mesenchyme in the limb (Fig. 2G; Fig. 5D) and parts of the diencephalon (Fig. 3A). In addition, the anti-teneurin-4 recog- nized the mesenchyme of the developing lung and showed teneurin-4 immunoreactivity in the apical ectodermal ridge (which was faintly, but not conclusively, labeled by in situ hybridization). In addition, the antiserum was used to study teneurin-4 in deeper tissues and at later stages of development not studied by in situ hybridization. For example, teneurin-4, unlike the other teneurins, is found in mesenchyme in the lung and gut. This expression is seen both at early and later stages of development.

Antibodies to teneurin-2 label some, but not all, basement membranes in the developing chicken (Tucker et al. 2001). Here, we show that an antibody to the extracellular EGF-like repeats of teneurin-4 also co-localize with an antibody to the laminin gamma 1 chain in some regions. This co-localization could be the result of extracellular teneurin-4 being shed and incorporated into the extracellular matrix, or it could be a reflection of the close proximity of membrane-bound teneurin- 4 with the basal lamina. In $C$. elegans, ten-1 null mutants have abnormal basement membranes, which appears to contribute to the abnormal development of the gut (Trzebiatowska et al. 2008); teneurin-4 may play a similar role in vertebrates.

The prominent expression of teneurin-4 in the limb led us to compare it with other teneurins. In comparison to the complex pattern of teneurin-4, teneurin-2 is exclusively expressed in the apical ectodermal ridge (see also Tucker et al. 2001) and teneurin3 is expressed in the dorsal, but not ventral, limb. In contrast to teneurin-4, which is mesenchymal in the distal anterior limb and epithelial in the distal posterior limb, teneurin-1 is mesenchymal in the ventral limb and is epithelial in the dorsal limb. This complicated pattern was observed with antisera raised against the ICD of teneurin-1 and against the EGF-like domains of teneurin-1, but the former was found in cell nuclei and the latter was not. The patterns of teneurin expression in the limb are intriguing given the known role for the Drosophila homolog ten ${ }^{\mathrm{m}}$ as a pair-rule gene, a family of genes encoding transcription factors that are responsible for the orderly segmentation of insect embryos. Baumgartner et al. (1994) found that $t e n^{\mathrm{m}}$ acts downstream of even skipped, epistatically to odd paired and upstream of engrailed and wingless. The vertebrate homologues of these genes have been shown to act in limb development as well. Evx1 (a homolog of even skipped) is first expressed in the distal mesenchyme of the posterior part of mouse limb bud (Niswander and Martin, 1993). Two vertebrate homologs of odd paired, Zic-2 and Zic-3, are also expressed in the distal limb bud mesenchyme, but Zic-3 is more abundant in the anterior mesenchyme where teneurin-4 is expressed (Nagai et al. 1997). Finally, both engrailed and a vertebrate wingless homolog, Wnt-7a, are expressed in the ventral and dorsal ectoderm of the limb buds, respectively (Parr et al. 1993; Logan et al. 1997). These limb expression patterns either match, or are complementary to, teneurin patterns of expression, just as they are in the parasegments of Drosophila. Thus, teneurins are likely to be working together with these factors to generate the complex epithelial-mesenchymal cross-talk necessary for the normal development of the limbs. The presence of teneurin-4 in the lung and gut is also consistent with a role in patterning and epithelial-mesenchymal interactions, not unlike those reported for sonic hedgehog, BMPs and Hox proteins (e.g., Roberts et al. 1998; Loscertales et al. 2008).

Teneurin-4, like each of the other teneurins, is expressed in the developing CNS. Each teneurin is expressed prominently in the 
visual system, where they partly overlap. Distinctive origins are reflected in part by their presence in different laminae in the inner plexiform layer of the retina, which are formed by dendritic arborizations from different classes of retinal ganglion cells. Though the retinas of many species are described as having five laminae within the inner plexiform layer, a detailed study of the chicken suggests that birds may have eight (Naito and Chen, 2004). Though our methods do not permit accurate mapping of the teneurins to each of these strata, in the central retina teneurin4 is most abundant in the deepest lamina (lamina 8), which lies adjacent to the inner nuclear layer, and is present faintly in two other strata in the outer half of the inner plexiform layer. This corresponds to the pattern of dendritic arborization of Class IVc retinal ganglion cells (Naito and Chen, 2004), which are characterized by their large cell bodies and extensive dendritic trees. The patterns seen with the other teneurin antisera were more complex and are probably a reflection of expression by multiple retinal ganglion cell classes.

Unlike the other teneurins studied to date, teneurin-4 is not expressed uniformly in the retina. This is first seen at E7, when teneurin-4 immunoreactivity is bright and limited to what appear to be bundles of axons in the optic fiber layer of the nasal retina. The bundling may be mediated in part by teneurin-4, as cells that express the ECD of teneurin-2 cluster in vitro (Rubin et al. 2002), and axons in $C$. elegans defasciculate following knockdown of ten-1 (Drabikowski et al. 2005). A few days later the part of the optic tectum to which these axons project is positive for teneurin4. This teneurin-4 immunostaining may be from the axons projecting from the nasal retina, the regional expression of teneurin-4 in the optic tectum, or both. At E12 the optic fiber layer of the nasal retina, but not temporal retina, remains positive for teneurin-4. Retinal ganglion cell bodies, however, are clearly immunostained in the temporal retina. At E17 the most prominent immunostaining is in the temporal retina, and the nasal retina is unstained. Thus, while teneurin-1 and teneurin-2 appear to be involved in establishing tectofugal and thalamofugal projections, respectively (Rubin et al. 2002), teneurin-4 may contribute to establishing connections that permit the mapping of the visual field.

Teneurin-4 is not only expressed in parts of the visual system, but also in parts of the limbic system (lateral habenular nucleus) and auditory system (the mesencephalicus lateralis pars dorsalis, nucleus laminaris and nucleus magnocelluaris). The labeling of the nucleus laminaris and nucleus magnocellularis is uneven, with the most lateral parts of these nuclei labeled strongly, but not central or medial regions. We showed previously that teneurin-2 is also present in the lateral-most part of the nucleus laminaris and that teneurin- 1 is present in the more medial portions, suggesting that teneurins may help establish gradients that map auditory inputs (Kenzelmann et al. 2008). Teneurin-4 may play a role in this mapping as well. It would be interesting to expand studies of teneurins to include expression within the cochlea, combined with tracing studies, to test this hypothesis.

The results of this study of avian teneurin- 4 are consistent with previous observations with other teneurins in other model organisms: teneurins appear to have phylogenetically conserved roles in axon bundling, pathfinding and patterning within the nervous system, and pattern formation in certain non-neuronal tissues during development. These many roles may be carried out by intact teneurins or teneurins that are processed so that $\mathrm{C}$-terminal peptides are released, the ECD is incorporated into the extracelIular matrix, and the ICD is free to regulate transcription. Future studies should be directed to understanding the mechanisms underlying fasciculation, the roles of teneurins in signaling during patterning, and the genes that are regulated by the ICD.

\section{Materials and Methods}

\section{Antibody production}

cDNA was made from RNA isolated from E17 chicken brains using oligo dT primers. To make an antibody against the ICD of teneurin-4, a $513 \mathrm{bp}$ PCR product corresponding to the first exon and first half of the second exon of predicted chicken teneurin-4 (NP 001092286; cten4 ICD) was amplified from the cDNA using the Hi-Fi Expand kit (Roche) and standard PCR conditions with primers

cten4fw (Sphl: 5'-ACTAGCATGCGATGTAAAGAAAGGAAACCG-3') and cten4rev (Kpnl: 5'-TAGTGGTACCAGTTTCGGTATTCTCGTGCTC-3'). The PCR product was cloned using the Sphl and Kpnl restriction sites into a PQE30 vector (Qiagen) containing a C-terminal His tag for protein expression and purification in bacteria. M15 Prep4 bacteria were transformed with the cten4 ICD construct and expression was confirmed by SDS-PAGE. After induction the soluble protein was purified from lysates under native conditions using a ProBond nickel column (Invitrogen) and eluted with increasing concentrations of imidazole (Fluka). The purified protein was injected into rabbits to generate polyclonal antibodies (antiteneurin-4 ICD), which were screened using blots of the expressed protein.

To make an antibody against the ECD of teneurin-4, a recombinant fusion protein between the chicken tenascin- $C$ signal peptide and the chicken teneurin-4 EGF-like repeats (TNC/cten4EGF) was generated by splicing by overlap extension. The tenascin-C fragment was produced from the pCTN-230 plasmid using the primers ( $5^{\prime}$ to $\left.3^{\prime}\right)$ :

$K S$ fW

CGAGGTCGACGGTATCG and

TNC/cten4EGFrev

ACAATTATCCACTGATTCATTGAGCCCAGTCTCCCG and the cten4EGF fragment using the primers

TNC/cten4EGFfw

CGGGAGACTGGGCTCAATGAATCAGTGGATAATTGT and cten4EGFrev

TAGTCTGGAGTTAGTGATGGTGATGGTGATGTCCATCGTTGTCCTTCCCGTC, which contained a His tag and an Xhol restriction site. To obtain the fusion protein, a second round of PCR was performed using both fragments as templates and the KSfw primer together with the cten4EGFrev primer. The resulting PCR product was cloned into the pCEP-Pu vector using the restriction enzymes Notl and Xhol and sequenced. Expression and secretion of the recombinant protein in 293T-EBNA cells was verified by immunoblot analysis of cell lysates and conditioned culture medium using the penta-His antibody (Qiagen). Cells stably expressing the TNC/ cten4EGF protein were selected with G418 (Promega) for the EBNA plasmid and puromycin (Sigma) for the pCEP plasmid. Cells were grown in $0.3 \%$ fetal calf serum in triple flasks (Nunc) to produce and secrete the TNC/cten4EGF protein. Subsequently, the recombinant protein was purified from conditioned medium and injected into rabbits to make the anti-teneurin-4 EGF serum.

\section{Immunoblotting and Immunohistochemistry}

Chicken embryos were sacrificed at E7 and E17 and the forebrain, retina, optic tectum and cerebellum were removed and homogenized in RIPA buffer (50mM Tris [Fluka], 150mM NaCl [Fluka], 0.1\% SDS [BioRad], $0.5 \%$ sodium deoxycholate [Merck] and $1 \%$ Triton X-100 [Fluka]). The homogenates were clarified by centrifugation and the protein concentration of the supernatant was determined with a Bradford Assay (BioRad). Equal amounts of protein were loaded onto a $7.5 \%$ polyacrylamide gel, 
separated by electrophoresis, and blotted for $2 \mathrm{hr}$ at $45 \mathrm{~mA}$ per gel using a semi-dry blotting system (Millipore). Equal loading and transfer were confirmed with amido black, then the blots were blocked with $5 \%$ milk (Fluka) and incubated overnight in anti-teneurin-4 ICD (1:1000) or antiteneurin-4 EGF (1:1000). The blots were then washed in Tris buffered saline with $0.1 \%$ Tween (Fluka), incubated in HRP-labeled secondary antibody (Cappel), and washed again. The antibody was detected using Super Signal (Pierce) and recorded on Hyperfilm (Amersham).

For immunohistochemistry, chicken embryos were sacrificed at E3.5, 4, 7, 10, 12 and 17, and appropriate regions (whole E3.5, 4 and 7; heads or wings E10,12 and 17; as well as just brains from E17) were fixed in ice cold $4 \%$ paraformaldehyde in phosphate buffered saline (PBS) overnight. Tissues were then rinsed in PBS and cryoprotected overnight in $20 \%$ sucrose in PBS. Tissues were embedded in O.C.T. (Sakura) and sections were cut at $12 \mu \mathrm{m}$ in a Microm HM560 cryostat. Sections were collected on presubbed slides (Fisher), air dried, then rinsed in PBS, blocked in $0.5 \%$ bovine serum albumin in PBS, and incubated overnight in antiteneurin-4 EGF (1:400). The teneurin-1 ICD and teneurin-1 EGF antisera were described previously (Kenzelmann et al. 2008), as was the antiteneurin-2 (Rubin et al. 1999). The teneurin-3 rabbit polyclonal antibody was raised against a teneurin-3 peptide sequence (VNSDTVPTHPVTVPA) by Invitrogen EvoQuest, and is being described elsewhere (Leachman and Tucker, in preparation). The laminin mouse monoclonal antibody (“31") recognizes the laminin gamma 1 chain (Bayne et al. 1984) and was obtained through the Developmental Studies Hybridoma Bank and the University of lowa.

Sections were then rinsed in PBS, incubated in secondary antibody (goat anti-rabbit Alexa 488 or Alexa 594; Molecular Probes), rinsed and coverslipped with Hoechst 33342 (Sigma) in the mounting medium. Controls were incubated in diluted preimmune serum or with secondary antibody alone. Sections were viewed and imaged on a Nikon Eclipse E800, Zeiss Z1 or a Zeiss Axioscop fluorescence microscope.

\section{Acknowledgements}

This work was funded in part by National Science Foundation grant 0235711 to R.P.T.

\section{References}

BAGUTTI, C., FORRO, G., FERRALLI, J., RUBIN, B. and CHIQUET-EHRISMANN, R. (2003). The intracellular domain of teneurin-2 has a nuclear function and represses zic-1-mediated transcription. J Cell Sci 116: 2957-2966.

BAUMGARTNER, S., MARTIN, D., HAGIOS, C. and CHIQUET-EHRISMANN, R. (1994). Tenm, a Drosophila gene related to tenascin, is a new pair-rule gene. EMBO J 13: 3728-3740.

BAYNE, E.K., ANDERSON, M.J. and FAMBROUGH, D.M. (1984). Extracellular matrix organization in developing muscle: correlation with acetylcholine receptor aggregates. J Cell Biol 99: 1486-1501.

DRABIKOWSKI, K., TRZEBIATOWSKA, A. and CHIQUET-EHRISMANN, R. (2005). ten-1, an essential gene for germ cell development, epidermal morphogenesis, gonad migration, and neuronal pathfinding in Caenorhabditis elegans. Dev Biol 282: 27-38.

FASCETTI, N. and BAUMGARTNER, S. (2002). Expression of Drosophila Ten-a, a dimeric receptor during embryonic development. Mech Dev 114: 197-200.

KENZELMANN, D., CHIQUET-EHRISMANN, R., LEACHMAN, N.T. and TUCKER, R.P. (2008). Teneurin-1 is expressed in interconnected regions of the developing brain and is processed in vivo. BMC Dev Biol 8: 30.

LEAMEY, C.A., MERLIN, S., LATTOUF, P., SAWATARI, A., ZHOU, X., DEMEL, N., GLENDINING, K.A., OOHASHI, T., SUR, M. and FÄSSLER, R. (2007). Ten_m3 regulates eye-specific patterning in the mammalian visual pathway and is required for binocular vision. PLOS Biol 5: e241.

LEVINE, A., BASHAN-AHREND, A., BUDAI-HADRIAN, O., GARTENBERG, D., MENASHEROW, S. and WIDES, R. (1994). Odd Oz: a novel Drosophila pair rule gene. Cell 77: 587-598.

LEVINE, A., WEISS, C. and WIDES, R. (1997). Expression of the pair-rule gene odd
Oz (odz) in imaginal tissues. Dev Dyn 209: 1-14.

LOGAN, C., HORNBRUCH, A., CAMPBELL, I. and LUMSDEN, A. (1997). The role of Engrailed in establishing the dorsoventral axis of the chick limb. Development 124: 2317-2324

LOSCERTALES, M., MIKELS, A.J., HU, J.K., DONAHOE, P.K. and ROBERTS, D.J. (2008). Chick pulmonary Wnt5a directs airway and vascular tubulogenesis. Development 135: 1365-1376

LOVEJOY, D.A., ROTZINGER, S. and BARSYTE-LOVEJOY, D. (2009). Evolution of complementary peptide systems: teneurin C-terminal-associated peptides and corticotropin-releasing factor superfamilies. Ann N Y Acad Sci. 1163: 215220.

MINET, A.D., RUBIN, B.P., TUCKER, R.P., BAUMGARTNER, S. and CHIQUETEHRISMANN, R. (1999). Teneurin-1, a vertebrate homologue of the Drosophila pair-rule gene ten-m, is a neuronal protein with a novel type of heparin-binding domain. J Cell Sci 112: 2019-2032.

NAGAI, T., ARUGA, J., TAKADA, S., GÜNTHER, T., SPÖRLE, R., SCHUGHART, K. and MIKOSHIBA, K. (1997). The expression of the mouse Zic1, Zic2, and Zic3 gene suggests an essential role for Zic genes in body pattern formation. Dev Biol 182: 299-313.

NAITO, J. and CHEN, Y. (2004). Morphologic analysis and classification of ganglion cells of the chick retina by intracellular injection of Lucifer Yellow and retrograde labeling with Dil. J Comp Neurol 469: 360-376.

NISWANDER, L. and MARTIN, G.R. (1993). FGF-4 regulates expression of Evx-1 in the developing mouse limb. Development 119: 287-294.

NUNES, S.M., FERRALLI, J., CHOI, K., BROWN-LUEDI, M., MINET, A.D. and CHIQUET-EHRISMANN, R. (2005). The intracellular domain of teneurin-1 interacts with MBD1 and CAP/ponsin resulting in subcellular codistribution and translocation to the nuclear matrix. Exp Cell Res 305: 122-132.

PARR, B.A., SHEA, M.J., VASSILEVA, G. and MCMAHON, A.P. (1993). Mouse Wnt genes exhibit discrete domains of expression in the early embryonic CNS and limb buds. Development 119: 247-261.

RAKOVITSKY, N., BUGANIM, Y., SWISSA, T., KINEL-TAHAN, Y., BRENNER, S. COHEN, M.A., LEVINE, A. and WIDES, R. (2007). Drosophila Ten-a is a maternal pair-rule and patterning gene. Mech Dev 124: 911-924.

RHOLAM, M. and FAHY, C. (2009). Processing of peptide and hormone precursors at the dibasic cleavage sites. Cell Mol Life Sci 66: 2075-2091.

ROBERTS, D.J., SMITH, D.M., GOFF, D.J. and TABIN, C.J. (1998). Epithelialmesenchymal signaling during the regionalization of the chick gut. Development 125: 2791-2801.

RUBIN, B.P., TUCKER, R.P., BROWN-LUEDI, M., MARTIN, D. and CHIQUETEHRISMANN, R. (2002). Teneurin 2 is expressed by the neurons of the thalamofugal visual system in situ and promotes homophilic cell-cell adhesion in vitro. Development 129: 4697-4705.

RUBIN, B.P., TUCKER, R.P., MARTIN, D. and CHIQUET-EHRISMANN, R. (1999) Teneurins: a novel family of neuronal cell surface proteins in vertebrates, homologous to the Drosophila pair-rule gene product Ten-m. Dev Biol216: 195209.

TÖMBÖL, T., ALPÁR, A., EYRE, M.D. and NÉMETH, A. (2006). Topographical organisation of projections from the nucleus isthmi magnocellularis to the optic tectum of the chick brain. Anat Embryol (Berl) 211: 119-128.

TRZEBIATOWSKA, A., TOPF, U., SAUDER, U., DRABIKOWSKI, K. and CHIQUETEHRISMANN, R. (2008). Caenorhabditis elegans teneurin, ten-1, is required for gonadal and pharyngeal basement membrane integrity and acts redundantly with integrin ina-1 and dystroglycan dgn-1. Mol Biol Cell 19: 3898-3908.

TUCKER, R.P., MARTIN, D., KOS, R., and CHIQUET-EHRISMANN, R. (2000). The expression of teneurin-4 in the avian embryo. Mech Dev 98: 187-191.

TUCKER, R.P., CHIQUET-EHRISMANN, R., CHEVRON, M.P., MARTIN, D., HALL, R.J. and RUBIN, B.P. (2001). Teneurin-2 is expressed in tissues that regulate limb and somite pattern formation and is induced in vitro and in situ by FGF8. Dev Dyn 220: 27-39.

TUCKER, R.P., KENZELMANN, D., TRZEBIATOWSKA, A. and CHIQUETEHRISMANN, R. (2007). Teneurins: transmembrane proteins with fundamental roles in development. Int J Biochem Cell Biol 39: 292-297.

YOUNG, T.R. and LEAMEY, C.A. (2009). Teneurins: important regulators of neural circuitry. Int J Biochem Cell Biol 41: 990-993. 
Further Related Reading, published previously in the Int. J. Dev. Biol.

See our recent Special Issue Placenta edited by Joan S. Hunt and Kent L. Thornburg at: http://www.ijdb.ehu.es/web/contents.php?vol=54\&issue=2-3

Molecular mechanisms controlling brain development: an overview of neuroepithelial secondary organizers

Claudia Vieira, Ana Pombero, Raquel García-Lopez, Lourdes Gimeno, Diego Echevarria and Salvador Martínez Int. J. Dev. Biol. (2010) 54: 7-20

Generation of pattern and form in the developing limb Matthew Towers and Cheryll Tickle Int. J. Dev. Biol. (2009) 53: 805-812

Limb pattern, physical mechanisms and morphological evolution - an interview with Stuart A. Newman Cheng-Ming Chuong Int. J. Dev. Biol. (2009) 53: 663-671

The Apical Ectodermal Ridge: morphological aspects and signaling pathways Marian Fernandez-Teran and Maria A. Ros Int. J. Dev. Biol. (2008) 52: 857-871

Conserved genetic mechanisms for embryonic brain patterning. Heinrich Reichert

Int. J. Dev. Biol. (2002) 46: 81-87

5 yr ISI Impact Factor $(2009)=3.253$
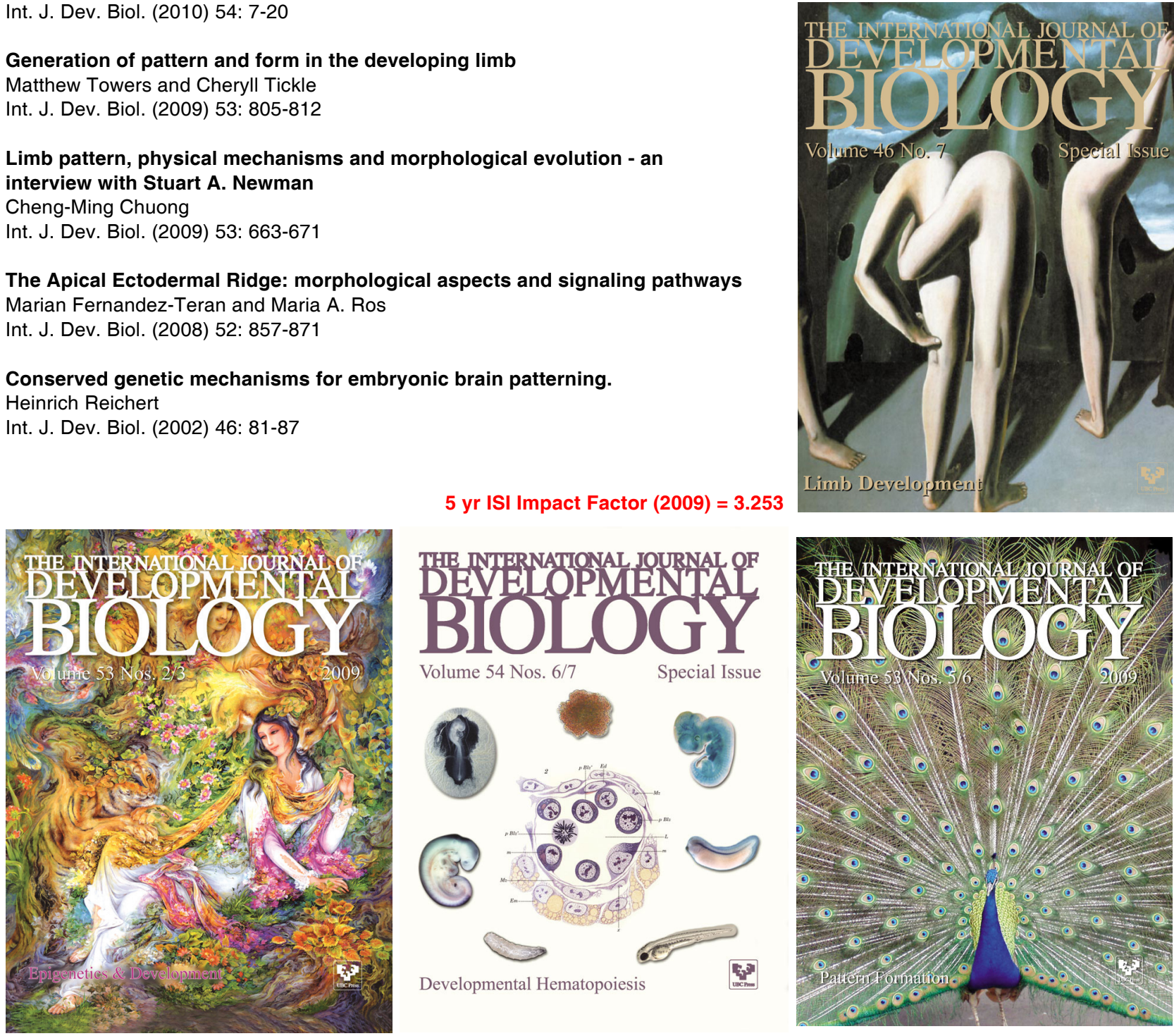

Volume 54 Nos. 6/7

Special Issue
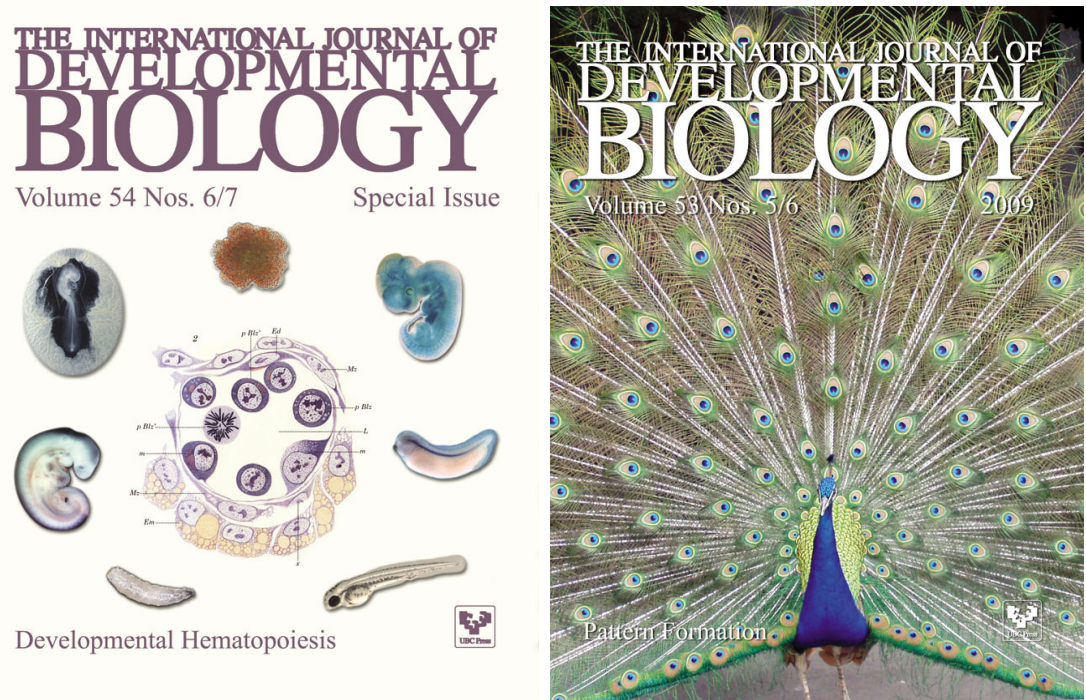

Developmental Hematopoiesis

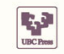

